\title{
Philosophiques
}

\section{Le bras long du sens commun}

\section{En guise de théorie de la méthode scientifique}

\section{Susan Haack}

Volume 30, numéro 2, automne 2003

URI : https://id.erudit.org/iderudit/008668ar

DOI : https://doi.org/10.7202/008668ar

Aller au sommaire du numéro

Éditeur(s)

Société de philosophie du Québec

ISSN

0316-2923 (imprimé)

1492-1391 (numérique)

Découvrir la revue

\section{Citer cet article}

Haack, S. (2003). Le bras long du sens commun : en guise de théorie de la méthode scientifique. Philosophiques, 30(2), 295-320.

https://doi.org/10.7202/008668ar d'utilisation que vous pouvez consulter en ligne.

https://apropos.erudit.org/fr/usagers/politique-dutilisation/ 


\title{
Le bras long du sens commun En guise de théorie de la méthode scientifique
}

\author{
SUSAN HAACK \\ University of Miami \\ s.haack@miami.edu
}

La méthode scientifique, dans la mesure où c'est une méthode, bouscule sans ménagement notre esprit, et tous les coups sont permis. (Percy Bridgman, "The Prospect for Intelligence », Yale Review. 1955)

Représentez-vous un scientifique comme quelqu'un qui travaille sur sa section dans une énorme grille de mots croisés: s'appuyant sur l'information dont il dispose, il devine la réponse, vérifiant encore et encore si celle-ci concorde avec l'indice et les entrées déjà complétées qui la croisent et si ces dernières concordent aussi avec leurs indices de même que les autres entrées, soupesant la probabilité que certaines de celles-ci soient erronées, puis essayant de nouvelles entrées à la lumière de celle-là, et ainsi de suite. La grille est en grande partie vide, mais beaucoup d'entrées sont déjà complétées, certaines à l'encre quasi indélébile, d'autres à l'encre ordinaire, d'autres encore au crayon plus ou moins appuyé, au point parfois de s'effacer. Certaines sont en anglais, d'autres en swahili, en flamand, en espéranto, etc., etc. Dans certaines sections, plusieurs longues entrées ont été écrites à l'encre d'une main ferme; ailleurs, il y en a peu ou pas. Certaines entrées ont été complétées des centaines d'années auparavant par des scientifiques morts depuis longtemps, d'autres la semaine dernière. À certaines époques, en certains lieux, sous peine de renvoi ou pire encore, seuls les mots du novlangue peuvent être utilisés; ailleurs, des pressions s'exercent pour que telles entrées soient remplies d'une certaine façon à l'exclusion d'une autre, ou pour qu'on se penche sur une section complètement vide plutôt que de travailler sur une partie plus facile et déjà partiellement remplie - ou pour qu'on ne travaille pas du tout sur certaines sections. Des équipes rivales se querellent au sujet de certaines entrées, les repassant au crayon ou même à l'encre puis gommant tout, peut-être dans une douzaine de langues et dans un délai déterminé. D’autres équipes coopèrent en vue de mettre au point une procédure pour débiter toutes les anagrammes d'un indice long comme un chapitre ou un appareil capable d'agrandir un indice si minuscule qu'il en est illisible, ou elles veulent lancer un appel aux équipes travaillant sur d'autres parties de la grille afin de voir si elles n'auraient pas quelque chose qui puisse être adapté ou pour demander si elles sont bien sûres qu'il faut mettre un «s» ici. Quelqu'un prétend avoir remarqué un détail dans tel ou tel indice que personne n'a 
jamais vu; d'autres conçoivent des tests pour vérifier si celui-ci est un observateur particulièrement talentueux ou s'il imagine des choses; d'autres encore travaillent pour mettre au point des instruments afin d'y voir de plus près. De temps en temps, des accusations sont portées au sujet d'indices qu'on aurait altérés ou de cases qu'on aurait noircies. Parfois, on entend ceux qui travaillent sur une partie de la grille se plaindre que leur point de vue sur ce qui se fait ailleurs n'est pas pris en compte. Ici et là, une longue entrée, qui en croise de nombreuses autres, est effacée par un groupe de jeunes Turcs qui affirment avec insistance que cette partie de la grille doit être refaite, et en turc cette fois, naturellement; d'autres encore tentent, lettre à lettre, de voir si le gallois original ne pourrait pas être préservé... Je ne cherche pas ici à vous refiler une métaphore en guise d'argument. Mais je cherche à suggérer, par cette histoire de mots croisés, que la quête scientifique est plus brouillonne, moins méthodique que les vieux déférencialistes ne l'imaginent, et pourtant davantage contrainte par les éléments de preuve que ne le pensent les nouveaux cyniques.

L'analogie de la grille de mots croisés s'est révélée un guide utile pour les questions d'éléments de preuve, mais d'aucuns pourraient avoir le sentiment qu'eu égard aux questions de méthode, elle est manifestement inutile. Après tout, que dire de la "méthode" de résolution des grilles de mots croisés, sinon que vous devez faire un pari sur certaines entrées à la lumière de l'indice qui lui est associé, essayer de voir les autres entrées à la lumière de leurs indices et des entrées déjà complétées puis, lorsqu'une entrée qui semble par ailleurs plausible s'avère incompatible avec les autres, que vous ne devez pas y renoncer trop facilement ni vous y accrocher de façon trop obstinée ? Et qu'est-ce que tout cela nous dit de la méthode "scientifique ", sinon que vous devez faire une conjecture informée au sujet de l'explication d'un phénomène qui vous intéresse, puis voir comment elle résiste aux éléments de preuve dont vous disposez et à ceux sur lesquels vous pourriez mettre la main, puis, lorsque votre conjecture qui apparaît autrement plausible s'avère incompatible avec certains éléments de preuve, que vous ne devez pas y renoncer trop facilement ni vous y accrocher trop obstinément ?

En effet, mais selon moi, l'analogie est utile. Elle nous guide vers la bonne conclusion: la "méthode scientifique» est quelque chose de moins formidable qu'il n'y paraît. La recherche scientifique appartient-elle à une autre catégorie que les autres types de recherches? Non. La recherche scientifique est en continuité avec la recherche empirique de tous les jours - c'est la même chose, mais à un degré supérieur. Y a-t-il un mode d'inférence ou une procédure de recherche auxquels tous les scientifiques et seulement eux ont recours? Non. Il y a seulement, d'une part, des modes d'inférence et des procédures utilisés par quiconque s'enquiert de ce qui est et, d'autre part, des techniques mathématiques, statistiques ou inférentielles spéciales, des instruments, des modèles spéciaux, etc., que l'on retrouve localement dans tel ou tel secteur de la science. Cela ébranle-t-il les 
prétentions épistémologiques de la science? Non! Les sciences naturelles sont épistémologiquement distinctes, elles ont remporté des succès remarquables, entre autres, précisément, grâce aux appareils et techniques au moyen desquels elles ont élargi la portée des méthodes dont use la recherche de tous les jours.

Nonobstant cet usage honorifique mais agaçant de «science» et autres termes apparentés, ce ne sont pas tous les scientifiques et seulement eux qui sont de bons chercheurs. Et il n'y a pas de procédure distincte ou de mode d'inférence utilisé par tous les praticiens de la science et seulement eux qui garantisse des résultats sinon vrais, au moins approximativement ou probablement vrais, ou plus proches de la vérité, ou empiriquement plus adéquats — pas de "méthode scientifique ", au sens où l'expression a souvent été comprise. La recherche en science est en continuité avec les autres types de recherches empiriques, mais les scientifiques ont conçu de nombreuses et diverses façons d'étendre et de raffiner les ressources auxquelles nous avons recours dans nos recherches empiriques de tous les jours.

Déjà dans Evidence and Inquiry, j’ai suggéré que les sciences, bien qu'épistémologiquement distinctes, ne sont pas privilégiées et "qu'il n'y a pas de raison de penser que [la science] soit en possession d'une méthode de recherche spéciale qui ne soit pas à la disposition des historiens, ou des détectives, ou du commun des mortels ${ }^{1}$ »; mais ces premiers et brefs efforts pour décrire la place des sciences naturelles au sein de la recherche empirique en général ont été poliment ignorés par la communauté des théoriciens de la science. Plus tard, je me suis souvenue de l'aphorisme de John Dewey selon lequel "la matière de et les procédures de la science sont issues des problèmes et des méthodes du sens commun ${ }^{2}$ ", ainsi que de l'ouvrage de James B. Conant, Science and Common Sense ${ }^{3}$. Puis, à ma surprise et à ma joie, j'ai trouvé cette remarque de Thomas Huxley disant que "l'homme de science utilise avec une exactitude scrupuleuse les méthodes que nous tous, par habitude et à chaque minute, utilisons sans y faire attention ${ }^{4} »$. Dans le même esprit, Albert Einstein a dit que «toute la

1. Susan Haack, Evidence and Inquiry. Towards Reconstruction in Epistemology, Oxford, Blackwell, 1993, p. 137. Peu après, j’ai développé ces idées dans le texte intitulé "Puzzling Out Science», Academic Questions, Spring 1995, p. 20-31 (repris dans Haack, Manifesto of a Passionate Moderate. Unfashionable Essays, Chicago, University of Chicago Press, 1998, p. 90-103), et dans "Science as Social? Yes and No", Feminism, Science, and Philosophy of Science (sous la dir. de J. Nelson et L. Hankinson Nelson), Dordrecht, Kluwer, 1996, p. 79-93 (repris dans Manifesto of a Passionate Moderate, p. 104-122).

2. John Dewey, Logic. The Theory of Inquiry, New York, Henry Holt, 1938, p. 66.

3. James B. Conant, Science and Common Sense, New Haven, Yale University Press, 1951.

4. Ma référence est J. W. Grove. In Defence of Science. Science, Technology and Politics in Modern Society, Toronto, University of Toronto Press, 1989, p. 13. Grove ne donne pas de référence exacte et j'ai été incapable de retrouver le passage en question. 
science n'est rien de plus qu'un raffinement de la pensée de tous les jours ${ }^{5}$ ", Percy Bridgman qu' «il n'y a pas de méthode scientifique comme telle, [...] l'aspect le plus vital de la procédure scientifique consiste simplement à tirer le meilleur parti des ressources de son esprit ${ }^{6} »$. Quant à Gustav Bergman, il a décrit les sciences, en une formule qui sonne merveilleusement bien, comme le «bras long du sens commun ${ }^{7}$ ». C'est dans cet esprit que je propose ici non pas une nouvelle théorie de la méthode scientifique, mais une exploration des contraintes pesant sur la recherche empirique quotidienne et des ressources qu'elle requiert, de la gamme étonnamment variée des voies et moyens que les sciences ont trouvés pour faire ce que fait la recherche de tous les jours «à un degré supérieur».

\section{1. «Rien d'autre qu'un raffinement de la pensée de tous les jours»}

Il y a "beaucoup de chichis autour de la méthode scientifique", écrit Bridgman, mais, poursuit-il, on fait beaucoup de bruit pour rien ${ }^{8}$. L'affaire des scientifiques est de chercher des réponses vraies à leurs questions; ainsi, comme pour toutes les recherches empiriques sérieuses, il leur faut vérifier les éléments de preuve aussi exhaustivement que possible et tenter de réfréner toute inclination à prendre leurs désirs pour la réalité. En outre, ils doivent avoir recours à toutes les méthodes spécifiques ou techniques concevables en rapport avec l'objet d'étude et ce qui en est connu, méthodes et techniques suceptibles de les aider à trouver une réponse aux questions qui les préoccupent ${ }^{9}$. Jusqu'ici, tout va bien. Mais de manière générale, quels sont les standards et les ressources de la recherche empirique bien conduite? Qu'est-ce que la recherche empirique bien conduite exige de ceux qui s'y livrent? Et en quoi, exactement, la recherche scientifique est-elle une recherche empirique, mais «à un degré supérieur»?

La recherche, à la différence de la composition d'une symphonie, de la préparation d'un repas, de l'écriture d'un roman, d'une plaidoirie devant la Cour suprême, constitue une tentative pour découvrir une réponse vraie à une ou plusieurs questions, bien que parfois le dénouement ne soit pas une réponse, mais plutôt la prise de conscience que la question était en quelque sorte mal formulée et que, bien souvent, lorsqu'une réponse à une question a été trouvée, toute une flopée de nouvelles questions font soudain

5. Albert Einstein, "Physics and Reality», The Journal of the Franklin Institute, vol. 221, $\mathrm{n}^{\circ}$ 3, 1936; repris dans Ideas and Opinions of Albert Einstein (sous la dir. de S. Bargmann), New York, Crown Publishers, p. 290.

6. Percy W. Brigman, "New Vistas for Intelligence», Physical Science and Human Values, Princeton, Princeton University Press, 1947; repris dans Reflections of a Physicist, p. 554.

7. Gustav Bergmann, Philosophy of Science, Madison, University of Wisconsin Press, p. 20 .

8. Percy W. Bridgman, "On "Scientific Method" ", The Teaching Scientist, décembre 1949; repris dans Reflections of a Physicist, p. 81.

9. Ibid., p. 81-83. 
surface. Il y a la recherche empirique et (du moins en apparence, mais ce n'est pas le lieu de s'aventurer dans des questions d'épistémologie des mathématiques et de la logique ${ }^{10}$ ) la recherche non empirique. La recherche empirique comprend tant les sciences naturelles, les sciences sociales, l'histoire, la recherche médico-légale, et ainsi de suite, que la recherche de tous les jours, quand vous vous demandez à quelle heure part votre avion, où acheter de la farine chappatie, comment imprimer en italique, ce que vous avez mangé qui vous a dérangé l'estomac, et ainsi de suite. Certaines recherches sont mieux menées que d'autres - de façon plus scrupuleuse, plus approfondie, plus imaginative, etc. - d'autres moins. Cela vaut pour tous les types de recherches, y compris la recherche scientifique.

Comme tous ceux qui font de la recherche empirique, les scientifiques veulent fournir une description qui soit vraie d'une certaine partie ou un certain aspect du monde. Toutes les descriptions vraies ne peuvent pas faire l'affaire, cependant; ainsi : "Ou bien le Big Bang est à l'origine de l'univers, ou bien il n'en est rien.» Il faut qu'une description ait de la substance, qu'elle soit significative, fournisse une véritable explication. Ce n'est pas une tâche facile. Tout ce qu'on a pour procéder, après tout, c'est ce qu'on voit, etc., et ce qu'on en comprend, etc. Parvenir à des conjectures explicatives plausibles peut pousser l'esprit humain jusqu'à sa limite; les éléments de preuve sont toujours incomplets et ramifiés, souvent potentiellement trompeurs et fréquemment ambigus; en obtenir davantage est un dur labeur et peut demander une ingéniosité considérable dans l'élaboration d'un dispositif expérimental. Et le succès n'est pas garanti, de sorte que la tentation de bâcler le travail est toujours là, comme celle de croire ce qu'on espère ou craint, ou ce qu'on pense que les commanditaires souhaitent entendre.

Bien que les théories scientifiques soient parfois étonnamment en porte-à-faux avec le sens commun, la recherche scientifique est en continuité avec la recherche empirique qui nous est la plus familière. Henry Harris imagine un peuple préhistorique qui tente de savoir si le fleuve qui coule à travers (ce que nous appelons) Oxford est le même que celui qui coule à travers Henley en faisant flotter des billots colorés dans le fleuve à partir d'Oxford et en demandant ensuite à des gens de leur connaissance à Henley s'ils les ont vus. Puis il décrit les efforts déployés par les physiologistes pour découvrir ce qui arrive à un grand nombre de lymphocytes qui passent de la lymphe au sang: un jour, J. L. Gowans a découvert, en les marquant avec des isotopes radioactifs, que les lymphocytes passent du sang à la lymphe pour retourner ensuite au sang ${ }^{11}$.

10. Voir Susan Haack, Deviant Logic, Fuzzy Logic. Beyond the Formalism, Chicago, University of Chicago Press, 1996.

11. Henry Harris, "Rationality in Science», Scientific Explanation (sous la dir. de A. F. Heath), Oxford, Clarendon Press, 1981, p. 40-44. 
Tous ceux qui font de la recherche empirique - les biologistes moléculaires et les musicologues, les entomologistes et les étymologistes, les sociologues et les théoriciens des cordes, les journalistes d'enquête et les immunologistes - font des conjectures informées sur une explication possible des phénomènes qui les intéressent, ils vérifient comment ces conjectures résistent aux éléments de preuve qu'ils ont déjà et à ceux sur lesquels ils mettent éventellement la main; ils se servent alors de leur jugement pour déterminer s'ils vont conserver ces conjectures, les rejeter, les modifier, etc. Il leur faut de l'imagination pour songer à des explications potentielles plausibles de phénomènes problématiques, pour concevoir des façons d'obtenir les éléments de preuve dont ils ont besoin et pour anticiper les sources d'erreurs potentielles; du soin, de l'habileté et de la persévérance pour chercher des éléments de preuve pertinents que personne n'a encore trouvés ou identifier ceux que d'autres ont déjà trouvés; de l'honnêteté intellectuelle et une solide fibre morale pour résister à la tentation d'ignorer des données qui pourraient affecter leurs hypothèses ou à celle de manipuler des données incontournables mais défavorables; un raisonnement rigoureux pour discerner les conséquences de ces conjectures; un bon jugement pour soupeser les divers éléments de preuve sans être perturbés ni par leurs désirs, leurs craintes ou leurs espoirs d'obtenir une permanence ou de résoudre rapidement un cas, de plaire à un patron ou à un mentor ou devenir riches et célèbres.

Faire une conjecture informée («informée» étant ici le mot clé) requiert la capacité de tirer les bonnes inférences: cette conjecture implique nos connaissances d'arrière-fond, cette autre est consistante avec elles, cette autre encore ne l'est pas. La vérification de la compatibilité de notre conjecture avec les éléments de preuve exige la même capacité: ainsi, si l'hypothèse est vraie, il s'ensuit telle conséquence; l'hypothèse est confirmée jusqu'à un certain point quand on se rend compte que telle conséquence est réalisée; elle a de bonnes chances d'être fausse si telle autre ne l'est pas, etc. Ce que je veux souligner ici, ce n'est pas que les scientifiques ne font pas de telles inférences ni que la logique n'a rien à nous apprendre à ce propos, mais plutôt que les détectives, les journalistes d'enquête, les historiens et le commun des mortels font aussi de telles inférences; la logique ne peut pas à elle seule expliquer comment les sciences ont connu de tels succès (encore moins pourquoi il y a autant d'échecs). La quête des vieux déférencialistes d'une méthode scientifique - logique inductive de la découverte ou de la confirmation, conjecture et réfutation-par-modus tollens du déductivisme poppérien, applications répétées du théorème de Bayes ou quoi encore se concentrait trop étroitement sur une partie seulement d'un portrait bien plus complexe.

Parmi les raisons qui expliquent le succès des sciences naturelles, il faudrait mentionner non pas précisément le hasard, mais le fait qu'il s'est trouvé quelques individus remarquables avec le tempérament et le talent 
pour spéculer sur la manière dont le monde fonctionne, et suffisamment d'autres individus pour se pencher sur la grille de mots croisés et vérifier si les réponses concordaient, et ce, en des lieux et des époques où le climat social et intellectuel leur permettait de poursuivre leurs recherches et de communiquer leurs résultats. Cela suggère que c'est à partir de là que nous devrions chercher l'explication de l'apparition de la science moderne pourquoi la science moderne est apparue au moment et à l'endroit où elle l'a fait, et non pas plus tôt ou ailleurs, l'expression qui vient à l'esprit étant celle de «masse critique».

Une autre raison à ce succès réside dans la matière même des sciences naturelles, dans ce profond caractère d'interconnexion des phénomènes naturels. C'est peut-être ce que E. O. Wilson a en tête lorsqu'il suggère que la méthode de la science est le "réductionnisme", soit une investigation systématique de la composition des choses en composantes de plus en plus petites. Mais une telle analyse, pour importante qu'elle soit, n'est qu'une approche scientifique parmi bien d'autres. À mon sens, la signification épistémologique de ce caractère d'interconnexion des phénomènes naturels réside plutôt dans ce que chaque avancée de la compréhension en rend d'autres possibles.

La formule qui vient à l'esprit est: «Le succès appelle le succès.» Cela nous amène à considérer les nombreuses aides à la recherche de divers types conçues par les scientifiques, aides sur lesquelles je me vais principalement me concentrer dans ce qui va suivre. Pour les fins de l'exposition, j'aurai recours à une division rudimentaire en termes d'aides à l'imagination, d'aides aux sens, d'aides au raisonnement, d'aides à la mise en commun des données et à l'honnêteté intellectuelle - bien que, puisque cellesci sont toutes liés aux visées de la recherche et aux capacités cognitives des chercheurs humains et à leurs limitations, elles soient en réalité intimement entrelacées.

\section{Les aides scientifiques à la recherche}

L'expression "aides» est empruntée à Francis Bacon, qui - en dépit de tous les espoirs mal avisés qu'il a fondés sur une logique de la découverte inductive et mécanique et de ce que, comme William Harvey l'a dit sans aménité, «il écrivait sur la science comme un chancelier» — voyait parfaitement juste lorsqu'il lançait des mises en garde contre le risque de désespérer trop vite que nos investigations puissent être couronnées de succès et insistait sur la nécessité de concevoir des moyens de surmonter nos limites sensorielles et cognitives et la fragilité de notre engagement à l'égard de la découverte $^{12}$. Comme Bacon l'avait bien compris, nous, êtres humains, sommes des créatures faillibles, notre imagination, nos sens et nos capacités cognitives sont limités, notre intégrité intellectuelle, fragile; la précipitation,

12. Francis Bacon, The New Organon (1620), aphorism 2. 
le travail bâclé et la propension à prendre nos désirs pour des réalités nous viennent plus facilement que la difficile et exigeante voie de la recherche consciencieuse, créative et honnête. Néanmoins, nous sommes capables de mener des recherches bien conduites et de concevoir des moyens, même imparfaits et limités, de surmonter nos limites et faiblesses naturelles, d'arriver à prédire quand nos moyens imparfaits et faillibles de surmonter ces limites et faiblesses sont le plus susceptibles de faillir, ainsi que de concevoir des façons d'éviter ces embûches.

Les exigences sous-jacentes et les ressources communes à toute recherche empirique sont constantes, mais les aides scientifiques évoluent constamment. Bien que certaines traversent tout le champ des sciences et même en débordent, beaucoup sont localisées, propres à certains secteurs de la science. Elles reposent généralement sur des travaux scientifiques antérieurs, et lorsque ceux-ci sont solides, elles permettent à la science de se bâtir, de s'édifier sur ses succès. Bien sûr, lorsque les travaux antérieurs sur lesquels les aides reposent ne sont pas solides, les scientifiques peuvent être induits en erreur - comme il arrive quand on remplit une grille de mots croisés.

Les modèles, métaphores et analogies qui aident l'imagination des scientifiques ont incité certains nouveaux cyniques à assimiler la science à la littérature et d'autres à déplorer que les métaphores et analogies se reflétant dans le contenu des théories scientifiques soient socialement rétrogrades; les instruments d'observation qui ajoutent aux pouvoirs perceptifs des scientifiques ont nourri l'idée que l'observation dépend par trop de la théorie pour procurer une vérification des données empiriques qui soit authentiquement objective; les conditions artificielles en laboratoire parfois nécessaires pour tester des affirmations théoriques en ont conduit certains à affirmer que les théories scientifiques décrivent non pas le monde naturel, mais seulement une "réalité » créée par les scientifiques eux-mêmes; sur la base des aspects sociaux de la recherche scientifique, d'aucuns ont conçu la connaissance scientifique comme une construction sociale au service des intérêts des puissants. Et certains nouveaux cyniques soutiennent que le caractère local, évolutif des aides scientifiques révèle que les standards de la recherche bien conduite sont relatifs à un contexte ou à un paradigme.

Mais ce sont toutes là des réactions excessives. Il est vrai que, parce qu'il y a toujours un risque que les travaux antérieurs sur lesquels reposent tel ou tel instrument, telle ou telle technique se révèlent erronés, le risque d'erreur est toujours possible. Certes, en jugeant si le travail a été conduit de façon consciencieuse et avec soin, les scientifiques doivent se baser sur ce qu'ils croient savoir des données susceptibles d'être pertinentes, des sources potentielles d'erreurs expérimentales, et ainsi de suite. La recherche scientifique est, en d'autres mots, faillible; les jugements sur la qualité de la recherche scientifique, comme les jugements sur la valeur des données empiriques, sont affaire de perspective et ils dépendent des connaissances 
d'arrière-fond. Mais il ne s'ensuit pas, contrairement à ce que Kuhn et d'autres semblent croire, qu'il faille en tirer des conclusions relativistes.

Toute recherche, recherche scientifique comprise, demande de l'imagination. Comme l'a affirmé Charles S. Peirce : «Lorsqu'un homme désire ardemment connaître la vérité, son premier effort va consister à imaginer ce que cette vérité peut être. [...] il n'y a rien d'autre, après tout, que l'imagination qui puisse jamais lui donner l'idée de ce qu'est la vérité.» Mais à la différence d'un artiste ou d'un écrivain, un scientifique "rêve d'explications et de lois ${ }^{13}$ » - explications et lois qui, lorsque ses efforts sont couronnés de succès, sont non pas imaginaires, telles des entités fictives, mais réelles.

Notre imagination, comme toutes nos facultés, est limitée; par conséquent, parmi les aides sur lesquelles tablent les scientifiques, il y a les modèles, analogies et métaphores. Dans son livre La Théorie physique, paru en 1914, Pierre Duhem a opposé l'esprit de géométrie, abstrait, logique, systématique, caractéristique à son avis des physiciens du continent, et la pensée visuelle, imaginative caractéristique de la pensée anglaise, qu'il considérait comme une diversion par rapport à l'abstraction mathématique au cœur même de la physique. À propos du livre sur l'électrostatique d'Oliver Lodge, Duhem déplorait : "Nous croyions pénétrer dans la demeure paisible et bien ordonnée de la raison, mais nous voilà au beau milieu d'une manufacture. » Car il n'y a rien dans le livre que des «cordes tournant autour de poulies, s'enroulant autour de tambours, passant à travers des perles [et] des roues dentées engrenées les unes aux autres et entrầnant des crochets ${ }^{14}$ ». Je vois d'ici Duhem s'arracher les cheveux quand John Kendrew invite ses lecteurs à imaginer "un homme [...] agrandi jusqu'à la taille $[. .$.$] du Royaume-Uni » et explique alors qu' "une seule cellule peut$ être aussi grande, peut-être, qu'une bâtisse d'usine. [...] À cette échelle, une molécule d'acide nucléique [...] serait plus mince qu'un seul filament d'ampoule électrique dans notre usine ${ }^{15}$ ». De nos jours, les étudiants en biologie sont parfois invités à concevoir la cellule comme une cité complexe, où les mitochondries sont des centrales électriques, les appareils de Golgi des bureaux de poste, et ainsi de suite. À la différence de Duhem, cependant, je considère qu'opposer les mathématiques à la construction de modèles, l'analyse à l'analogie, la systématisation à la simulation, c'est créer de

13. Charles Sanders Peirce, Collected Papers (texte établi par C. Hartshorn, P. Weiss et A. Burks), Cambridge, Mass., Harvard University Press, 1931-1958, vol. I, $\mathbb{\$}$ 46-8.

14. Pierre Duhem, La Théorie physique: son objet, sa structure, 1914; cité par Mary Hesse, Models and Analogies in Science, Londres, Sheed and Ward, 1962, p. 1-2. (Faute d'avoir retrouvé la citation originale, ce passage, comme tous les autres tirés de l'ouvrage de Duhem, sont des retraductions de l'anglais. N.D.T.) 
fausses dichotomies; les modèles, analogies et métaphores jouent un rôle important non pas seulement d'un point de vue pédagogique, mais aussi au niveau de la construction de théories, comme des aides à l'imagination.

«Les modèles, analogies et métaphores» forment une catégorie hétéroclite comprenant de tout, depuis les arrangements physiques concrets de billes, de boules ou de maquettes, etc., jusqu'aux métaphores maîtresses comme la «main invisible» dans la théorie des marchés d'Adam Smith; tous, cependant, comparent un phénomène moins familier ou accessible à un autre qui l'est davantage. L'un des rôles des modèles physiques, comme la série de maquettes de la molécule d'ADN de Watson et Crick, est d'aider visuellement l'imagination, permettant aux scientifiques d'appréhender la molécule, à l'instar du modèle, en trois dimensions. Et si certaines métaphores scientifiques sont plus décoratives que fonctionnelles, d'autres suggèrent des questions à investiguer, des directions à explorer. Bien entendu, une métaphore peut pousser différents scientifiques à regarder dans différentes directions; parfois, elle peut tirer la recherche vers ce qui se révèle une mauvaise direction.

Comme les critiques de la science se plaisent à le souligner, les métaphores et analogies que les scientifiques utilisent s'inspirent parfois de phénomènes sociaux familiers; ainsi la métaphore de la "molécule maîtresse ", qu'Evelyn Fox Keller a enrôlée dans la cause féministe (à ce que je sache, cependant, cette idée n'a jamais, contrairement à ce qu'elle a suggéré, eu une grande influence en biologie moléculaire). Les métaphores scientifiques peuvent être cognitivement importantes, et elles peuvent aussi bien induire l'imagination en erreur que la guider dans des directions fructueuses. Mais le caractère fructueux ou non d'une métaphore ne dépend pas de la désirabilité ou non du phénomène social auquel elle fait appel; de telles considérations ne déterminent en rien si les notions de chaperons moléculaires (pour utiliser un exemple dont l'influence est réelle ${ }^{16}$ ), ou d'investissement parental, etc., ou quoi encore, nous conduiront vers des territoires fertiles ou pas.

Toute recherche empirique dépend de l'expérience; mais si la recherche en sciences constitue "la même chose mais à un degré supérieur", on peut mentionner que c'est entre autres parce que l'expérience dont elle dépend,

15. John Kendrew, The Thread of Life, Cambridge, Mass., Harvard University Press, 1966, p. 20.

16. Voir Elizabeth Craig, "Chaperones, Molecular", Molecular Biology and Biotechnology (sous la dir. de A. Meyers), New York, VCH Publishers, 1995, p. 162-165: "Les chaperons moléculaires sont des protéines omniprésentes qui jouent un rôle capital dans le processus d'enveloppement des protéines et le déplacement des protéines à travers les membranes vers les organelles.» 
loin de se passer de toute assistance, est rendue possible et médiatisée par des instruments de toutes sortes; elle n'est pas non plus invérifiée, mais est ouverte à l'examen rigoureux des autres membres de la communauté; et elle n'est pas laissée à la chance ou à quelques heureux hasards, mais elle est délibérée, contrainte, contrôlée.

Je commencerai par les instruments d'observation, des plus familiers, comme le microscope ou le télescope, aux plus sophistiqués et ésotériques, qui prolongent maintenant les pouvoirs sensoriels des scientifiques. Dans l'histoire de tels instruments, il n'y a sans doute pas de cas plus saisissant, qui fasse dire que "le succès appelle le succès ", que celui de l'imagerie médicale. Wilhelm Roentgen a réalisé la première photographie aux rayons X en 1895 (en 1901, il recevait le tout premier prix Nobel). La première radiographie dentaire a été faite en 1896, le premier diagnostic basé sur les rayons X posé sur le champ de bataille en Abyssinie en 1897. Cette annéelà, les rayons $\mathrm{X}$ ont été utilisés pour la première fois en cour. Dans les décennies qui ont suivi, on a assisté au développement de la théorie des rayons $\mathrm{X}$ - soit les très courtes ondes électromagnétiques, dont la longueur d'ondes est de 0,10 à 10 nanomètres. Entre 1919 et 1927, des milieux de contraste, d'abord l'air, puis les lipoïdes, puis l'iodure de sodium, puis le dioxyde de thorium, ont rendu les photographies aux rayons $\mathrm{X}$ plus informatives. Le tomographe, qui produit l'image d'une coupe interne du patient, a été introduit par Jean Keiffer dans les années 1930, la cristallographie par rayons X est apparue à la même époque. À partir des années 1970, des machines à imagerie beaucoup plus rapides ont été développées, et maintenant, avec la technologie sophistiquée des ordinateurs, nous avons le tomographe assisté par ordinateur (CAT), l'IRM, qui permet de faire disparaître les os et de révéler les tissus, le tomographe par émission de positrons, qui utilise des traceurs radioactifs, et des instruments pour détecter ces traces et créer des images, puis le tomographe $\mathrm{EBT}^{17}$.

La théorie à la base d'un instrument pourrait se révéler erronée, peutêtre au point d'ébranler la confiance des scientifiques dans le fonctionnement de cet instrument; mais si la théorie est solidement justifiée, ce scénario, bien que possible, est peu probable. L'instrumentation dépend d'une théorie, mais ce qui explique le fonctionnement d'un instrument est rarement, sinon jamais, la théorie dont les conséquences sont testées par l'instrument, théorie que les observations tirées de l'instrument doit alors confirmer. Ainsi, les scientifiques se tournent vers l'optique pour rendre compte

17. Le CAT est un tomographe assisté par ordinateur (ou axial), l'IRM correspond à l'«imagerie par résonance magnétique " (le magnétisme est à l'IRM ce que les radiations sont aux rayons $\mathrm{X}$ ); le PET au tomographe à émission de positrons et l'EBT au tomographe à faisceau d'électrons. Ma source est l'histoire de la technologie de l'imagerie médicale par Bettyann Holtzmann Kevles, Naked to the Bone. Medical Imaging in the Twentieth Century, New Brunswick, Rutgers University Press, 1997. Au sujet de l'EBT, voir Tara Parker-Pope, "New Tests Go Beyond Cholesterol to Find Heart-Disease Risks", Wall Street Journal, 22 juin 2001, p. B1. 
du fonctionnement du microscope grâce auquel ils étudient la constitution des cellules, ou encore celui du télescope qui leur permet d'étudier le mouvement des étoiles. Il est vrai que les théories scientifiques s'entrecroisent, telles les entrées d'une grille de mots croisés, si bien que, par conséquent, la possibilité lointaine d'une dépendance mutuelle préjudiciable ne peut pas tout à fait, en principe, être écartée. Il est vrai aussi que, particulièrement dans les premiers temps, les scientifiques ont parfois eu besoin d'entrer dans les moindres détails pour persuader les autres de la fiabilité de leurs instruments. Certes, les scientifiques prennent parfois des artifices dus à leurs instruments pour des éléments de preuve en bonne et due forme (de l'avis de certains, c'est ce qui s'est produit dans le cas des preuves de vie bactérienne sur Mars). Mais rien de ceci ne signifie que les instruments ne se sont pas, en général, des aides véritables.

Les chercheurs sérieux, dans quelque domaine que ce soit, s'engagent activement dans la quête de preuves: les historiens recherchent des documents, interrogent les survivants, et ainsi de suite; les détectives suivent et observent les suspects, et ainsi de suite. Mais la recherche en sciences naturelles est souvent une recherche "à un degré supérieur ", dans la mesure où elle implique souvent la manipulation des conditions qui vont rendre disponible tel ou tel élément de preuve. Les chercheurs scientifiques mettent toute leur ardeur à concevoir des expériences qui seront aussi informatives que possible, isolant précisément la variable qui les intéresse - comme lorsqu'on travaille sur les entrées croisant telle autre précisément là où les cases admettent des solutions différentes. Les expériences d'Oswald Avery en vue d'identifier la substance responsable de la "transformation bactérienne " fournissent un exemple frappant de la finesse et de la connaissance des faits qu'une bonne conception expérimentale requiert.

Chez des souris qui avaient reçu une injection d'une préparation de pneumocoques de type $\mathrm{R}$ vivants mais non virulents et de pneumocoques de type $S$ morts mais virulents, Frederick Griffith a découvert des pneumocoques de type $S$ vivants et virulents. Plus tard, des collaborateurs d'Avery ont découvert que les mêmes transformations bactériennes pouvaient être produites in vitro. Pour découvrir ce qui était responsable de celles-ci, Avery a d'abord élaboré un processus sophistiqué pour extraire le "principe de transformation", quel qu'il fût, soit moins d'une once sur vingt gallons de culture. Il a ensuite soumis cet extrait à des tests standards pour les protéines, avec des résultats négatifs, puis à des tests standards pour l'ADN, avec des résultats fortement positifs. Grâce à l'analyse chimique, il a découvert que l'extrait contenait le ratio azote-phosphore de 1,67/1 que l'on attend de l'ADN, mais pas des protéines. Puis, il a découvert que les enzymes reconnues pour dégrader les protéines ou l'ARN laissaient l'extrait intact, mais que celles reconnues pour dégrader l'ADN le détruisaient. 
Ensuite, en ayant recours à des tests immunologiques, il a découvert que ni la protéine pneumococcale ni la capsule de polysaccharide n'étaient présents. Il a fait tournoyer un extrait de l'échantillon dans une centrifugeuse à très haute vitesse puis a découvert une structure de sédimentation correspondant à l'ADN du thymus du veau. Enfin, il a découvert que sous électrophorèse, les molécules de l'extrait demeuraient ensemble et se déplaçaient assez rapidement, à l'instar des acides nucléiques, et que le profil d'absorption des ultraviolets de l'extrait était le même que celui de l'ADN.

Dans une lettre à son frère, Avery écrivait: "La substance est hautement réactive et se conforme très étroitement aux valeurs théoriques de l'acide désoxyribonucléique pur [...] Qui aurait pu le deviner ${ }^{18}$ ?» (En raison de l'influence de l'hypothèse des tetranucléotides, toutefois, il a pris soin d'indiquer en conclusion de l'article qu'il a publié qu'il n'excluait pas que le principe de transformation soit non pas l'ADN lui-même, mais une infime quantité de quelque chose d'autre absorbé par l'ADN.)

Songeons maintenant à la multiplicité des moyens pris afin de s'assurer que les expériences et les observations ne sont pas contaminées, physiquement ou autrement, des plus banals, tels l'interdiction d'apporter de la nourriture dans le laboratoire où se déroulent des expériences biochimiques, les listes de vérifications pour s'assurer systématiquement du bon fonctionnement d'un équipement complexe et les formulaires standardisés sur lesquels les observations sont notées de façon à ce qu'aucun détail pertinent ne soit oublié, aux plus sophistiqués, comme le double aveugle, en passant par les plus difficiles, par exemple le choix des procédures d'échantillonnage les plus appropriés eu égard à la question en jeu, procédures qui pourraient biaiser les résultats.

Qu'il soit approprié de prendre telles précautions plutôt que telles autres - interdire la nourriture ou les animaux dans le labo, disons, mais pas les souliers et les stylos à bille - dépend de certains présupposés concernant le genre de choses susceptibles d'interférer. Il est concevable que ces présupposés soient erronés, et il est parfois très difficile de les découvrir. Ainsi, parmi les aspects de la manière dont l'expérimentateur conduit tel test psychologique ou les faits concernant l'expérimentateur luimême, lesquels sont susceptibles d'affecter la réponse? Lorsque je critique la conception d'une étude psychologique où les sujets ont été prévenus que le but des entrevues auxquelles ils participeraient était d'identifier leur "mode féminin de connaître ", je tiens pour acquis que cette suggestion rend probable un éventuel biais des résultats ${ }^{19}$. On peut concevoir (à la

18. Ma référence est Horace Freeland Judson, The Eighth Day of Creation. Makers of the Revolution in Biology, New York, Simon and Schuster, 1979, p. 35-39.

19. Mary Field Belenky et coll., Women's Ways of Knowing, New York, Basic Books, 1986; Susan Haack, "Knowledge and Propaganda: Reflections of an Old Feminist ", Partisan Review, vol. X no 4, automne 1993, p. 556-563, repris dans Manifesto of a Passionate Moderate, p. 123-136, p. 125. 
rigueur) que je sois dans l'erreur. Mais que les précautions prises ne soient pas infaillibles ne signifie pas qu'elles ne sont pas, de manière générale, de véritables aides.

Lorsque nous critiquons des recherches piètrement menées, nous pouvons déplorer que l'on n'ait pas fait d'efforts suffisants pour mettre la main sur des éléments de preuve pertinents; ainsi, d'un détective paresseux, on dira: "Il ne s'est même pas donné la peine de retracer la bonne pour lui demander ce qu'elle avait vu.» Ou nous pouvons déplorer que l'on n'ait pas pris suffisamment soin d'établir la valeur de certains éléments; d'un historien brouillon - et cette fois, le brouhaha soulevé par une lettre montrant prétendument que Marilyn Monroe a fait chanter le président Kennedy est un exemple réel ${ }^{20}-$, on dira: "Il a sauté à la conclusion que la lettre était authentique, ignorant le fait que l'adresse comporte un code postal et que du ruban correcteur a été utilisé alors que ni l'un ni l'autre n'existaient au moment où la lettre est censée avoir été écrite. » Et ainsi de suite.

Des reproches du même genre valent également pour la recherche scientifique; mais dans ce cas, nous déplorons, et à juste titre, que certaines précautions appropriées et spécifiques à un certain domaine n'aient pas été prises. Comme le remarque Bridgman, "[lorsque] le scientifique se risque à critiquer le travail de ses collègues scientifiques, comme il n'est pas rare de le faire, il ne base pas ces critiques sur de brillantes généralités concernant le non-respect de la "méthode scientifique", mais sa critique est spécifique, basée sur certains aspects de la situation particulière ${ }^{21}$ ». Les critiques d'une étude établissant prétendument l'efficacité médicale de la prière, par exemple, ont objecté que celle-ci n'était pas complètement à la doubleaveugle $^{22}$; Maddox et compagnie ont fait la même objection concernant le travail du labo de Benveniste établissant prétendument l'efficacité des remèdes homéopathiques à haute dilution: "aucun effort substantiel n'a été fait pour exclure l'erreur systématique, y compris le biais de l'observateur », et «le phénomène décrit n'est pas reproductible au sens ordinaire du terme». Il est toutefois assez intéressant de remarquer qu'une pensée très humienne sous-tend ces deux critiques: l'idée que des prières faites en faveur d'un patient mais à son insu puisse contribuer à sa guérison est étrangère à la science médicale; celle qu'une "solution» à ce point diluée qu'elle ne contient plus une seule molécule du supposé «soluté » agisse parce qu'elle laisse des traces dans la «mémoire » de l'eau est profondément

20. Evan Thomas, Mark Hosenball et Michael Isikoff, "The JFK-Marilyn Hoax", Newsweek, June 6 ${ }^{\text {th }}, 1997$, p. 36-37.

21. Bridgman, "On "Scientific Method" ", p. 81.

22. Irwin Tessman et Jack Tessman, «Efficacy of Prayer: A Critical Assessment of Claims ", Skeptical Inquirer, March/April 2000, p. 31-33, critiquant R. C. Byrd, "Positive Therapeutic Effects of Intercessory Prayer in a Coronary Care Unit ", Southern Medical Journal, 1988, n 81 , p. 826-828. 
en désaccord avec toute la théorie chimique admise, au point qu'il y a beaucoup plus de chances que les découvertes alléguées soient le résultat d'erreurs expérimentales ou de méprises de la part de l'expérimentateur que d'authentiques découvertes ${ }^{23}$.

Investiguer notre prédisposition à l'erreur sous tel ou tel rapport nous rend plus aptes à comprendre quels résultats risquent d'être attribuables à des erreurs expérimentales ou à des erreurs d'autres types. Par exemple, la détection d'une fraude dans la recherche scientifique est souvent déclenchée par quelqu'un qui remarque que certains résultats sont «trop beaux pour être vrais ", confirmant une hypothèse avec une netteté supérieure à celle que suggère ce que nous savons de la faillibilité humaine, ou de celle des instruments.

En évoquant la conception des expériences et les précautions contre l'erreur expérimentale, j'ai déjà commencé à me hasarder dans le domaine des aides à intellect, mais jusqu'ici, j'ai à peine effleuré les nombreux raffinements et amplifications du raisonnement sur lesquels les sciences s'appuient.

Un des aspects les plus saisissants d'une grande partie de la recherche en sciences naturelles tient à son caractère mathématique. En effet, la naissance de la science moderne peut être attribuée à l'union des mathématiques et de la physique (anticipée par Archimède, sérieusement entreprise par Galilée, que Gillespie devait qualifier de meilleur élève d'Archimède, et perfectionnée avec Newton ${ }^{24}$ ). Il suffit de se rappeler qu'il n'y a pas si longtemps, le mot anglais computer référait non pas aux machines auxquelles Alan Turing ne pouvait que rêver, mais aux femmes employées pour calculer la trajectoire des pièces d'artillerie ${ }^{25}$, pour apprécier le rôle des mathématiques dans l'évolution des aides scientifiques: depuis le comptage au moyen d'entailles sur des bouts de bois ou de nœuds sur un bout de corde en passant par les chiffres romains puis arabes, jusqu'au calcul différentiel et intégral et maintenant l'ordinateur.

Déjà au moment de la découverte de la structure de l'ADN par Watson et Crick, le travail en biologie devenait presque aussi mathématique qu'en physique ou en chimie. De nos jours, les calculs compliqués sont

23. Jacques Benveniste et coll., "Human Basophil Degranulation Triggered by Very Dilute Antiserum Against IgE ", Nature, $n^{\circ} 330$, June 30 $0^{\text {th }}, 1998$, p. 816-818; John Maddox et coll., " "High-Dilution” Experiments a Delusion ", Nature, 1988, n 334, p. 287-290 (les citations proviennent de la page 287).

24. Charles Coulson Gillepsie, The Edge of Objectivity. An Essay in the History of Scientific Ideas, Princeton, Princeton University Press, 1960, p. 16, 144.

25. Voir Caroline Bird, Enterprising Women, New York, Mentor Books, W. W. Norton, 1976, p. 117. 
faits beaucoup plus rapidement et beaucoup plus précisément par les ordinateurs que par les humains; la presse nous informe du marathon de calcul étalé sur un mois au cours duquel les physiciens du Brookhaven National Laboratory ont calculé le moment angulaire anormal du muon et de la vaste opération de super-calcul grâce à laquelle Celera Genomics a produit sa carte du génome humain. À leur répertoire familier d'expériences in vivo (sur des créatures vivantes) et in vitro (dans des éprouvettes ou des vases de Petri), les biologistes ont maintenant ajouté les expériences in silica, c'est-à-dire simulées par ordinateur ${ }^{26}$.

Mais l'ordinateur est seulement l'exemple le plus évident d'une large catégorie d' "aides au raisonnement». Les diverses techniques encore controversées de méta-analyse, qui combinent tout un ensemble d'études épidémiologiques ou autres pour en extraire plus d'information qu'aucune d'elles ne pourrait en produire seule, en sont un autre. Dans un article récent fascinant, par exemple, deux chercheurs danois ont analysé de nouveau les données concernant l' "effet placebo ", dont l'existence a été tenue pour acquise depuis 1955, alors que Henry Beecher rapportait que 35 pour cent des patients étaient aidés par des placebos. Certains médecins ont maintenant recours aux placebos comme traitement, et certains chercheurs en médecine consacrent leur temps à comprendre comment l'effet placebo pourrait opérer. Mais si les docteurs Hrobjartsson et Gotzsche ont raison, cet effet supposé est largement mythique. À partir de 727 essais potentiellement admissibles, Hrobjartsson et Gotzsche ont réanalysé 114 études (impliquant en tout 7500 patients atteints de 40 affections différentes) qu'ils ont jugées "bien conçues »; les patients étaient divisés entre: 1) ceux qui recevaient des traitements médicaux réels; 2) ceux qui recevaient un placebo; 3) ceux qui ne recevaient rien du tout. À l'exception d'un effet léger au niveau de la réduction de la douleur, ils ont conclu qu'il y a peu de preuves que les placebos aient des effets cliniques significatifs ${ }^{27}$.

Ont-ils raison? Il est trop tôt pour en être sûr. Mais le travail de ces scientifiques et les réactions d'autres scientifiques illustrent de façon frappante la finesse requise par de tels raffinements méta-analytiques du raisonnement et leur dépendance à l'endroit de la connaissance des faits. Hrobjartsson et Gotzsche critiquent les méthodes de recherche de Beecher en faisant valoir qu'elles ne pouvaient pas adéquatement distinguer les

26. George Johnson, "All Science is Computer Science", New York Times, March $25^{\text {th }}, 2001$, section 4 , p. 1,5 .

27. Asbjorn Hrobjartsson et Peter Gotzsche, «Is the Placebo Powerless? An Analysis of Clinical Trials Comparing Placebo With No Treatment ", New England Journal of Medicine, vol. 344, $\mathrm{n}^{\circ} 21$, May 24 ${ }^{\text {th }}$, 2001, p. 1594-1602. Voir aussi John C. Bailar III, "The Powerful Placebo and the Wizard of $\mathrm{Oz}$ ", New England Journal of Medicine, ibid., p. 16301632; Gina Kolata, "Placebo Effect is More Myth Than Science, Study Says ", New York Times, May $24^{\text {th }}, 2001$, p. A 20, et «Putting Your Faith in Science? ", New York Times, May $27^{\text {th }}, 2001$, Section 4, p. 2. 
effets des placebos du cours naturel des maladies, la régression à la moyenne et autres facteurs (par exemple l'automédication par les patients, la possibilité que certains "placebos" ne soient pas entièrement inactifs après tout). Mais ils ont aussi reconnu que leurs propres méthodes pouvaient avoir besoin de plus de raffinement, observant que divers types de biais pouvaient avoir affecté leurs propres découvertes, en ce qui concerne les résultats subjectivement rapportés, en particulier.

Le caractère social de la recherche scientifique, qui est parfois vu comme une menace à ses prétentions épistémiques, serait mieux conçu comme un autre type d'aide faillible. Un grand nombre de tâches sont mieux effectuées si plusieurs personnes sont impliquées, mais le travail scientifique ne ressemble guère ni au catapultage de cent livres de petits pois - plus il y a de gens, plus cela se fait vite - , ni au transport d'un billot très lourd, qui peut être soulevé par plusieurs personnes mais non par une seule. Ce travail est complexe, intriqué et comporte de multiples facettes comme - eh oui! - le travail sur une vaste grille de mots croisés.

La justification est affaire de degré; les degrés de justification de théories rivales, qui plus est, ne seront pas nécessairement ordonnés de façon linéaire. Dans cette vaste zone grise où une attitude plus ou moins optimiste vis-à-vis d'une affirmation quelque-peu-mais-pas-totalement justifiée est raisonnable, il n'y a pas d'étape simple qui permette de passer des degrés de justification à des «règles d'acceptation et de rejet». Une conjecture pour le moment plus ou moins développée pourrait valoir la peine d'être explorée bien qu'elle soit, jusqu'ici, non justifiée; «la » meilleure façon de travailler consiste souvent à laisser différents membres de la communauté travailler de manières différentes.

Comme l'a observé Duhem, face à un problème non résolu, les sciences se trouvent souvent dans un état d'indécision entre des approches plus audacieuses et d'autres plus prudentes ${ }^{28}$. Certains d'entre nous sont prompts à gommer une entrée dans une grille de mots croisés lorsque les contraintes qu'elle impose sur les autres entrées commencent à nous gêner, d'autres sont plus lents à changer quelque chose. Si nous travaillions ensemble, je vous empêcherais peut-être de vous cramponner trop longtemps à une idée fixe, et vous m'empêcheriez peut-être de renoncer trop facilement à une idée prometteuse. Cela suggère que la recherche scientifique tend à mieux se porter lorsque la communauté comprend, comme c'est généralement le cas, certains individus prompts à se lancer dans des conjectures sur de nouvelles théories possibles lorsque les éléments de preuve commencent à desservir l'opinion reçue, et d'autres qui sont plus enclins à tenter de modifier avec patience ce dont ils disposent déjà.

28. Pierre Duhem, La Théorie physique: son objet, sa structure, p. 217. 
Michael Polyani a déjà souligné ce point important: bien que la meilleure façon d'organiser une armée d'écosseurs de pois pourrait être de confier à une seule personne la direction des opérations, pour la recherche scientifique, ce serait inapproprié, en raison même de sa complexité, de même que ce le serait pour une équipe travaillant sur un puzzle géant ${ }^{29}-$ ou, pour reprendre mon propre exemple, non le sien, sur une vaste grille de mots croisés. Comme Polyani l'a bien vu, il vaut mieux que différentes personnes, chacune avec son tempérament plus ou moins conservateur, ses forces et ses faiblesses, fassent ce qu'elles font le mieux. Parmi les nombreux et divers talents qui sont utiles en science, c'est l'extraordinaire créativité intellectuelle qui a permis aux héros de l'histoire des sciences de faire leurs étonnantes percées théoriques qui vient en premier lieu à l'esprit. Mais la liste est longue et diverse, comprenant entre autres ce don spécial pour discerner les motifs récurrents dans ce qu'ils voient qui échoit à certains scientifiques, comme, semble-t-il, le talent musical échoit à d'autres; l'ingéniosité dans la conception d'expériences ou dans l'invention d'instruments, de tests ou de modèles mathématiques; la simple patience dans le laborieux processus de la vérification et de la revérification.

C'est ici que les questions de communication, d'expertise et d'autorité deviennent notre point de mire. Si on ne veut pas dilapider ses forces en refaisant ce qui a déjà été fait, le travail de chacun doit être librement et convenablement mis à la desposition des autres. Et les scientifiques ont besoin, non seulement d'être en mesure de regarder par-dessus l'épaule des autres alors qu'ils travaillent, mais aussi de pouvoir se jucher sur les épaules de ceux qui sont passés avant eux, car il faudrait à chaque fois tout reprendre de zéro si on ne pouvait tenir les résultats précédents pour acquis. Les problèmes liés à la communication, à la transmission des résultats recoupent ceux relatifs à l'expertise et l'autorité. Des journaux encombrés d'articles écrits par des loufoques et des illuminés rendraient la recherche plus difficile, non pas plus facile. D'où le besoin d'avoir des moyens de distinguer le cinglé et l'incompétent du chercheur compétent - recommandations, évaluations par les pairs - de façon à s'assurer que ce que les journaux diffusent n'est pas de la camelote mais des travaux valables.

Comme les précautions prises contre la contamination dans les laboratoires et ainsi de suite, ces mécanismes sont faillibles. Des évaluateurs pas tout à fait honnêtes peuvent être tentés d'empêcher la publication des travaux de leurs rivaux; des évaluateurs pas tout à fait qualifiés peuvent manquer des connaissances préalables requises pour faire une évaluation juste; des évaluateurs sans imagination peuvent être incapables d'apprécier des idées vraiment innovatrices. Il $\mathrm{y} a$, de plus, un spectre très large entre

29. Michael Polanyi, The Republic of Science, Chicago, Roosevelt University, 1962; aussi dans Knowing and Being (sous la dir. de M. Grene), Chicago, University of Chicago Press, 1969, p. 49-62. 
la conjecture scientifique hétérodoxe mais créatrice, et la pathologiquement cinglée; une idée tordue ne se présente pas nécessairement avec la mention "désespérée », une idée créatrice et hétérodoxe avec la mention "prometteuse». Ce qui fait qu'un travail est jugé valable ou pas doit dépendre de ce qui est tenu pour des faits connus. Ainsi, Martin Gardner écrit, estimant qu'Immanuel Velikovsky dépasse vraiment les bornes, que ses idées, "si elles étaient correctes, demanderaient qu'on récrive la physique, l'astronomie, la géologie et l'histoire de l'Antiquitée ${ }^{30}$ ». Ce qui est tenu pour des faits connus est parfois faux - l'œuvre de Darwin, après tout, a exigé que l'on récrive une grande partie de ce qu'on croyait savoir des fossiles - , ces jugements peuvent donc être erronés. Mais comme pour les précautions contre la contamination expérimentale dont il a été question plus tôt, que ces précautions soient faillibles ne signifie pas qu'elles ne sont pas, en général, de véritables aides.

Nous devons dépasser l'idée que "plusieurs mains allègent la tâche" pour en venir à comprendre que la recherche implique une division du travail d'un (ou plusieurs) genres des plus subtils; dépasser l'idée que la science est affaire de "confiance " pour en venir à mettre au jour le délicat équilibre entre, d'une part, la critique mutuelle et la vérification institutionnalisées et, d'autre part, l'autorité institutionnalisée des résultats bien justifiés auxquels est parvenue la meilleure recherche qui soit en sciences naturelles. Les scientifiques comptent sur la compétence et l'expertise de chacun; mais, comme notre choix de mots l'indique, la confiance et l'autorité impliquées ne sont pas l'apanage de certaines personnes ou même de certaines positions, mais des distinctions qui se méritent.

À cette image des scientifiques qui regardent par-dessus les épaules les uns des autres, ou qui se juchent sur les épaules de ceux qui les ont précédés, je dois ajouter, puisque la recherche dans les sciences peut être affaire de compétition autant que de coopération, que l'on voit parfois des scientifiques se frayer un chemin à coups de coudes. (Je fais de mon mieux, mais brosser le tableau complet exige vraiment les talents d'un Brueghel!) Bien que ce ne soit pas invariablement ou nécessairement le cas, la compétition entre personnes ou équipes rivales peut être productive: l'espoir de battre les autres types pour obtenir le prix Nobel peut donner lieu à des miracles d'efforts intellectuels; les tenants d'une approche ou d'une théorie sont motivés à traquer les éléments de preuve susceptibles d'être défavorables à leurs rivaux, éléments que ceux-ci sont tentés de négliger ou de minimiser. La compétition peut être conçue comme un autre exemple d'aide; non pas, comme le microscope, une aide aux sens limités des êtres humains, ni, comme une analogie bien choisie, une aide à nos imaginations limitées, mais une aide à notre énergie limitée et à notre fragile intégrité intellectuelle.

30. Martin Gardner, Science. Good, Bad and Bogus, Buffalo, Prometheus Books, 1986, p. 235. 
Comme les autres, les aides sociales sont faillibles. Ainsi que le suggère le côté ouï-dire de la littérature scientifique sur l'effet placebo, avec ses couches sur couches de références croisées dans une multitude d'articles scientifiques qui remontent tous à l'unique étude insatisfaisante de Beecher, la confiance des scientifiques est parfois mal placée. La compétition peut mener à un gaspillage de ressources, même à la suppression d'un travail valable; la coopération peut prolonger une recherche inutile. Mais "faire l'impossible avec son propre esprit ", c'est aussi tenter de comprendre dans quelles circonstances les instruments ou la pratique de citations sont susceptibles de nous égarer, dans quelles circonstances la compétition est susceptible de tourner au vinaigre et d'être contre-productive, dans quelles circonstances la coopération est susceptible de dégénérer en expansionnisme bureaucratique, en travail inutile ou en promotion mutuelle. Et si on peut comprendre cela, on peut concevoir des garde-fous - imparfaits et faillibles, comme toujours, mais néanmoins utiles - contre le manque de précautions.

Les aides scientifiques sont souvent locales. (Un physicien n'a pas à se soucier, contrairement à un biochimiste, des animaux dans son laboratoire — en tout cas pas pour les mêmes raisons!) Néanmoins, la recherche scientifique et les autres genres de recherches sont désormais entrelacés de toutes sortes de manières, et les aides scientifiques sont aussi utilisées par d'autres. Les détectives comptent sur les techniques de la science médicolégale comme le typage du sang, les empreintes génétiques et le reste. Les historiens de l'Antiquité ont récemment commencé à se servir de techniques conçues pour la détection du cancer du sein pour déchiffrer les «cartes postales » de plomb que les soldats romains envoyaient chez eux ${ }^{31}$. D'autres historiens ont eu recours à l'analyse au neutron pour montrer que des morceaux de jaspe retrouvés sur un site dans le nord de Terre-Neuve contenaient des traces d'éléments présents seulement dans le jaspe du Groenland et d'Islande, confirmant ainsi la conjecture voulant que les Vikings auraient atteint l'Amérique du Nord un demi-millénaire avant Christophe Colomb ${ }^{32}$. General Motors se sert de techniques statistiques développées par les centres d'épidémiologie pour détecter des "épidémies " potentielles de défectuosités dans leurs voitures et camions ${ }^{33}$. Et ainsi de suite. À l'occasion, bien que ce soit moins fréquent, les scientifiques empruntent aux autres chercheurs, lorsque, par exemple, un arbre généalogique compilé par le père d'une personne souffrante apporte un indice

31. «Wish You Were Here", Oxford Today, vol. 10, n 3, Trinity 1998, p. 40.

32. Sharon Begley, "The Ancient Mariners", Newsweek, April 3 ${ }^{\text {rd }}$, 2000, p. 48-54, p. 54 .

33. Gregory L. White, "GM Takes Advice From Disease Sleuths to Debug Cars", Wall Street Journal, April $8^{\text {th }}, 1999$, p. B4. 
vital pour l'identification d'un défaut génétique responsable de la pancréatite héréditaire ${ }^{34}$.

\section{Réévaluation de l'ancien déférencialisme et du néo-cynisme}

En retournant aux vieux déférencialistes et aux nouveaux cyniques, un merveilleux poème pour enfants me revient à l'esprit, Les avengles et l'éléphant. Un Hindou aveugle, avançant à tâtons à côté d'un éléphant, décrète qu'un éléphant "ressemble fort à un mur ", un autre, s'emparant de sa queue, qu'un éléphant "ressemble fort à une corde », etc.:

Et ainsi des hommes de l'Hindoustan

Ont discuté, fort et longtemps

Chacun défendant sa propre opinion

Se surpassant en entêtement et en conviction

Bien que chacun eût en partie raison

Et que tous fussent dans l'erreur ${ }^{35}$

Les vieux déférencialistes ont, avec raison, tenu pour acquis que les sciences naturelles ont connu des succès éclatants. Mais ils se sont trompés dans la mesure où ils ont supposé que l'explication de ces succès reposait sur une méthode de recherche de nature étroitement logique ou quasi logique, propre à la science, qui garantissait sinon le succès, du moins le progrès. Les nouveaux cyniques sont à juste titre sceptiques vis-à-vis de l'existence de la "méthode scientifique", du moins au sens espéré; et ils voient bien qu'en se concentrant de façon trop exclusive sur les aspects étroitement logiques de la science, on passe outre le fait que, quoi qu'elle puisse être par ailleurs, la science est une institution sociale. Mais ils ont tort lorsqu'ils concluent qu'il est illusoire de penser que la recherche scientifique est de quelque façon épistémologiquement éminente. Plutôt que d'insister davantage sur cette idée générale, permettez-moi de l'appliquer, à la lumière de la conception qui vient d'être décrite ici, à certains vieux débats concernant le problème de la démarcation et la distinction entre découverte et justification.

Pour les vieux déférencialistes - pour les poppériens en particulier, mais aussi pour les positivistes, quoique de façon différente - , la distinction entre science et non-science était une préoccupation importante, confortant l'usage honorifique du mot "science» et le mythe d'une méthode scientifique caractéristiquement rationnelle et confortée par eux ${ }^{36}$. Mais si

34 Wendy Bounds, "One Family's Search for a Faulty Gene», Wall Street Journal, August $15^{\text {th }}$, 1996, p. B1, B7.

35. John Godfrey Saxe, "The Blind Men and the Elephant ", The Oxford Treasury of Children's Poems (sous la de M. Harrison et Ch. Stuart-Clark), Oxford University Press, Oxford, 1988, p. 116-117.

36. Bien sûr, le problème de démarcation remonte, sous une forme légèrement différente, à Platon et Aristote. Larry Laudan, "The Demise of the Demarcation Problem ", 
les vieux déférencialistes se préoccupaient trop de la question de la démarcation, les nouveaux cyniques (qu'ils pensent que la science n'est qu'une grande et puissante institution sociale parmi d'autres, ou qu'une création de l'imagination parmi d'autres, pas essentiellement différente de la fiction) écartent trop facilement du revers de la main les prétentions épistémologiques des sciences. Considérant l'usage honorifique de la mention "scientifique " comme une nuisance, et sceptiques à l'endroit de la «méthode scientifique» au sens déférencialiste du terme, je ne suis pas portée à accorder au "problème de la démarcation » la même importance que certains vieux déférencialistes. Mais, considérant que le terme "science" désigne une fédération de genres de recherche épistémologiquement éminents, j'accorde aux enjeux épistémologiques une place bien plus centrale que les nouveaux cyniques.

La première chose à dire est que la «non-science» est une catégorie vaste et diverse, comprenant les nombreuses autres activités qui ne sont pas de l'ordre de la recherche, les diverses formes de pseudo-recherches, la recherche de caractère non empirique et la recherche empirique d'autres genres que la recherche scientifique; pour rendre les choses encore plus compliquées, il y a encore un tas de cas limites et hybrides. L'usage du terme "scientifique» et des termes apparentés pour vanter des recherches empiriques de tous ordres incite les scientifiques comme les profanes (et les juges - mais je ne vais pas me lancer dans des problèmes d'ordre légal ici $^{37}$ ) à accuser la science piètrement conduite de ne pas être de la science du tout. Mais en fait de critique épistémique d'ordre général, "non scientifique» est aussi peu éclairant que qualifier de «scientifique» tout ce qu'on veut louanger.

L'expression "pseudo-science ", qui est censée référer aux activités se présentant comme des sciences mais qui n'en sont pas, mérite une attention particulière. Son caractère péjoratif dérive en partie de ce qu'on lui impute de fausses prétentions, mais aussi en partie des connotations honorifiques de «scientifique». Écoutons Bridgman de nouveau: «[Le] scientifique à l'œuvre se soucie toujours beaucoup trop des détails importants pour vouloir perdre son temps à des généralités ${ }^{38}$.» À mes yeux aussi, au lieu de reprocher à un travail d'être "pseudo scientifique", il vaut toujours mieux spécifier ce qui, exactement, ne va pas: que ce n'est pas une recherche honnête ou sérieuse; qu'elle repose sur des présupposés mal étayés ou bien

Physics, Philosophy, and Psychoanalysis (sous la dir. de R. S. Cohen et L. Laudan), Dordrecht, Reidel, 1983, p. 11-27 (repris dans Michael Ruse, dir., But is it Science? The Philosophical Question in the Creation/Evolution Controversy, Buffalo, Prometheus Books, 1996, p. 337350 ), est utile à la fois pour le versant historique et pour la problématique liée à ces questions.

37. Voir à ce sujet Susan Haack, "An Epistemologist in the Bramble Bush: At the Supreme Court With Mr. Joiner ", Journal of Health Politics, Policy and Law, vol. 26, $\mathrm{n}^{\circ} 2$, avril 2001, p. 217-248.

38. Bridgeman, «On Scientific Method», p. 81. 
trop vagues pour pouvoir être corroborés; qu'elle utilise un symbolisme mathématique, ou peut-être un appareillage en apparence très élaboré, purement décoratif; etc.

Lakatos s'inquiète de ce que "si Kuhn a raison, il n'y a pas de démarcation explicite entre science et pseudo-science, pas de distinction entre progrès scientifique et déclin intellectuel, il n'y a pas de standard objectif d'honnêteté intellectuelle [...] Quels critères peut-il alors offrir pour établir la démarcation entre progrès scientifique et dégénérescence intellectuell $^{39}$ ? ». En quelques phrases seulement, Lakatos invoque simultanément la science, le progrès scientifique, l'honnêteté intellectuelle et la santé intellectuelle d'une culture - qui sont tous des concepts bien distincts, quoique interreliés de façon subtile et complexe. En particulier, être prêt à reconnaître des éléments de preuve négatifs n'est pas un critère de la science authentique; c'est une condition de l'honnêteté intellectuelle — pour les scientifiques comme pour les chercheurs de tous genres. Darwin écrit qu'il a toujours suivi « une règle d'or, à savoir que chaque fois qu'un fait publié, une nouvelle observation ou une pensée me tombait dessus, qui s'opposait à mes résultats généraux, d'en prendre note invariablement et tout de suite; car l'expérience m'a appris que ces faits et pensées étaient beaucoup plus susceptibles d'échapper à la mémoire que ceux qui sont favorables ${ }^{40}$ ", expérience qui est familière à quiconque - scientifique, historien, journaliste, ou même philosophe - s'est déjà engagé dans une recherche sérieuse.

Bien sûr, à certaines fins, il est parfois nécessaire de tracer une ligne rudimentaire entre la science et les autres choses. Une façon de faire pourrait consister à penser que les sciences diffèrent d'activités telles que la danse folklorique ou l'art de plaider en ce que ce sont des genres de recherche; qu'elles diffèrent d'autres genres de recherche empirique comme l'histoire ou l'érudition légale ou littéraire en vertu de leur sujet; et peutêtre, qu'elles diffèrent de la théologie naturelle en vertu du genre d'explications qu'elles admettent. Aussi sommaire que cela puisse être, ce n'est pas un mauvais point de départ pour une explication de la manière dont, disons, la science diffère de la littérature ou de l'industrie du divertissement. Mais si nous voulons comprendre les aspects historiques de la science sociale, de la biologie évolutionniste ou de la cosmologie, tout en évitant d'assimiler la science à l'histoire, nous aurons besoin de quelque chose de plus subtil. Et si nous voulons comprendre comment le créationnisme diffère épistémologiquement de la cosmologie ou de la biologie évolutionniste, nous ferions mieux de nous concentrer directement sur des questions

39. Imre Lakatos, The Methodology of Scientific Research Programme (sous la dir. de J. Worral et G. Currie), Cambridge, Cambridge University Press, 1978, p. 4.

40. Charles Darwin, Autobiography and Letters (textes choisis par F. Darwin), New York, D. Appleton and Company, 1893, p. 45. 
concernant les éléments de preuve et la justification, au lieu de faire tout un plat sur la question de savoir si le créationnisme est de la mauvaise science, ou pas de la science du tout.

Revenons à la distinction découverte/justification. Les déférencialistes anciens et modernes ont espéré confiner les aspects sociaux et psychologiques de la science au contexte de la découverte et se concentrer sur le joli, logique et bien ordonné contexte de la justification ${ }^{41}$. Mais les nouveaux cyniques, naturellement, contestent la légitimité de la distinction. Toute cette affaire a encouragé à la fois la tendance déférencialiste à simplifier indûment le processus de la recherche et l'empressement des cyniques à ignorer le rôle des éléments de preuve.

Le problème est moins qu'il n'y a pas de distinction à tirer du contraste entre la découverte et la justification, mais qu'il y en a beaucoup trop. Il y a certainement une différence entre la question de savoir comment on est venu à une théorie et celle de savoir ce que valent les éléments de preuve pertinents. Il y a certainement différentes étapes de la recherche: une théorie est conçue, développée, testée, raffinée, modifiée, présentée dans les revues, etc. Et il y a certainement une différence entre les questions d'ordre psychologique et social concernant la recherche scientifique et celles d'ordre épistémologique. Mais identifier le contexte de justification à l'étape de présentation de la recherche, comme Reichenbach, c'est risquer de confondre la question de la qualité des éléments de preuve et celle de savoir ce que fait un scientifique pour persuader ses collègues de la véracité de sa théorie. Et identifier le contexte de la découverte à la sphère du psychologique et du sociologique et le contexte de la justification à la sphère de la logique, comme Popper, c'est risquer de travestir le fait que parvenir à une hypothèse ne se fait généralement pas à l'aveuglette, mais implique des inférences, et que recueillir, partager et soupeser les éléments de preuve pertinents à l'évaluation d'une hypothèse est généralement une entreprise collective.

Comme pour le problème de la démarcation, je voudrais proposer de mettre l'accent ailleurs, cette fois sur le rôle de l'inférence dans le processus par lequel les scientifiques arrivent à des théories, et sur celui des interactions entre les scientifiques lorsqu'ils évaluent les éléments de preuve. Aucune règle d'inférence ne garantit une bonne conjecture, mais une bonne

41. Karl R. Popper, The Logic of Scientific Discovery (d'abord paru en allemand en 1934), Londres, Hutchinson, 1959, chap. 1, $\mathbb{2}$; Hans Reichenbach, Experience and Prediction. An Analysis of the Foundations and the Structure of Knowledge, Chicago, University of Chicago Press, 1938, pp. 6-7. Paul Hoyningen-Huene, "Context of Discovery and Context of Justification", Studies in the History and Philosophy of Science, vol. 18, $\mathrm{n}^{\circ}$ 4, 1987, p. 501-515, présente une histoire de la distinction (dont une forme remonte au moins jusqu’à J. F. W. Herschel, Preliminary Discourse on the Study of Natural Philosophy, Londres, Longman, 1830-1831, p. 164); et une décomposition des différentes distinctions qu'il perçoit comme étant étroitement associées. 
conjecture doit être compatible avec ce qui est déjà connu (et si possible l'impliquer). Songez à quel point les contraintes étaient fortes pour les solutions possibles au problème de la structure de l'ADN, étant donné le vaste réseau de croyances de fond sur lequel Crick et Watson s'appuyaient, et les photographies de Franklin de la forme B. Watson est tombé sur la nature exacte des paires de base "par un heureux hasard ", rappelle Crick, mais on aurait pu y arriver par élimination, en testant systématiquement les paires suggérées par les règles de Chargaff ${ }^{42}$. Et, de même que quelque chose d'inférentiel est impliqué lorsque l'on aboutit à une conjecture plausible, de même quelque chose de social - l'interaction de membres plus ou moins conservateurs de la communauté scientifique pendant que les éléments de preuve nécessaires pour trancher entre une conjecture et ses rivales sont cherchés et triés - intervient dans le processus par lequel le résultat en vient éventuellement à être mentionné dans les ouvrages de référence ou bien rejeté.

\section{Et en conclusion}

Commentant l'article récent du New England Journal, le docteur Clement McDonald, qui dix-huit ans plus tôt publiait un article remettant en question l'authenticité de l'effet placebo, observe que «ce qu'il y a de bien avec la science, c'est que tôt ou tard, la vérité sort au grand jour ${ }^{43}{ }^{\text {». Sans doute }}$ que ceci semble un peu naï; néanmoins, de façon grossière, cette affirmation rend compte de quelque chose d'important. Le progrès a été réduit en lambeaux; toutefois, grâce aux sciences naturelles, nous en savons bien plus aujourd'hui sur le monde qu'il y a, disons, quatre cents ans.

Si l'histoire de l'ADN illustre ce qui fait la gloire de la science, la saga du météorite martien en illustre la décrépitude. 1996: les scientifiques suggèrent que les carbonates que le météorite dégage sont l'indice d'une vie bactérienne primitive sur Mars; 1997: d'autres scientifiques suggèrent des explications rivales à la présence de carbonates; 1998 : d'autres proposent que ces traces bactériennes sont dues à une contamination terrestre; 1999: des photographies prises par satellite indiquent qu'il pourrait y avoir de l'eau sous le permafrost martien, et le vaisseau spatial Polar Lander est envoyé à la recherche de preuves supplémentaires; janvier 2000: on pense que le Lander a été perdu quand il s'est écrasé dans un canyon sur Mars; février 2000: on suppose que certains signaux mystérieux pourraient être émis par le Lander après tout; avril 2000: les chercheurs de la NASA

42. Francis Crick, What Mad Pursuti. A Personal View of Scientific Discovery, New York, Basic Books, 1988, p. 65-66; voir Paul E. Meehl, "Corroboration and Verisimilitude: Against Lakatos's "Sheer Leap of Faith" ", Minnesota Center for Philosophy of Science, $\mathrm{n}^{\circ}$ 90-01, 1990, 1-49, p. 27.

43. Kolata, "Putting Your Faith In Science?». 
pensent que le Lander s'est écrasé sur la surface gelée de Mars et s'est fracassé ${ }^{44}$. Malgré tout, je ne doute pas que la vérité sortira un jour.

Laissez-moi suggérer une réinterprétation amicale de l'observation de Lakatos selon laquelle «il n'y a pas de rationalité instantanée », et de la conception de l'histoire de la science comme étant «écrite par le côté gagnant » de Kuhn. Le progrès peut être modeste et avancer à petits pas, ou grand et révolutionnaire, ou n'importe où entre les deux. Il peut résulter d'un accident heureux voire d'une erreur féconde, comme il arrive lorsqu'un scientifique, par ignorance ou par confusion, formule une hypothèse incompatible avec ce qui est tenu pour un fait connu, mais n'en est pas un. À certaines époques et à certains endroits, la recherche scientifique stagne ou même régresse, et ce n'est qu'avec le recul qu'il devient clair que tel ou tel changement était progressif. Néanmoins, si j'ai raison, il n’y a rien de mystérieux à ce que, dans l'ensemble, et dans une perspective à long terme, la recherche en sciences naturelles ait progressé. Car elle repose sur des aides qui, bien que faillibles et imparfaites, tendent généralement à aider l'imagination, à étendre l'accès aux données empiriques, et à consolider le respect pour les données. Tous les pas n'iront pas dans la bonne direction, loin de là, mais dans la mesure où les aides réussissent, la tendance générale ira dans la direction d'un ancrage plus solide dans l'expérience et d'une intégration explicative plus grande.

44. Adam Rogers, "Come in, Mars", Newsweek, October $20^{\text {th }}, 1996$, p. 56-57; Sharon Begley et Adam Rogers, "War of the Worlds", Newsweek, February 10 ${ }^{\text {th }}, 1997$, p. 56-58; "Meteorite - or Wrong?", Newsweek, January 26 ${ }^{\text {th }}, 1998$, p. 8; John Noble Wilford, " 2 New Chemical Studies Show No Traces of Life on Mars", New York Times, January $16^{\text {th }}$, 1998, p. A22; Thomas Hayden, "A Message, But Still No Answer », Newsweek, December $6^{\text {th }}, 1999$, p. 60; "NASA Scientists seem close to confirming...", Wall Street Journal, December $19^{\text {th }}, 1999$, p. A1; "Did the Mars Lander Crash in a Grand Canyon?", Newsweek, January $17^{\text {th }}$, 2000, p. 57; William Broad, "Evidence Builds that Mars Lander is Source of Mystery Signal», New York Times, February 1\$t, 2000, p. A17; Andrew Murr, «Final Answer: It Crashed», Newsweek, April 10" 2000. 